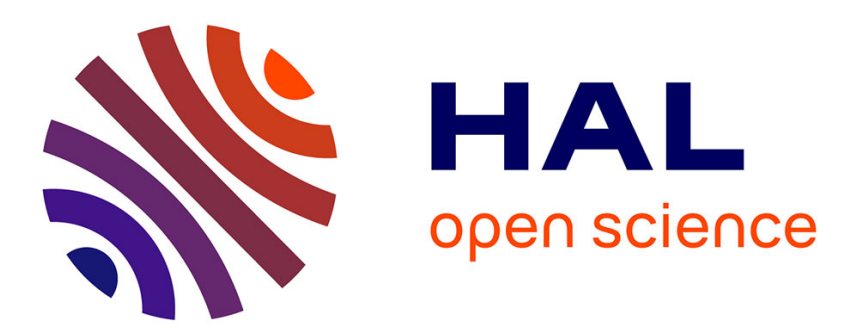

\title{
Localization of sources of ELF/VLF hiss observed in the magnetosphere: Three-dimensional ray tracing
}

\author{
L. Cairó, François Lefeuvre
}

\section{To cite this version:}

L. Cairó, François Lefeuvre. Localization of sources of ELF/VLF hiss observed in the magnetosphere: Three-dimensional ray tracing. Journal of Geophysical Research, 1986, 91 (A4), pp.4352. 10.1029/JA091iA04p04352 . insu-03124917

\section{HAL Id: insu-03124917 https://hal-insu.archives-ouvertes.fr/insu-03124917}

Submitted on 29 Jan 2021

HAL is a multi-disciplinary open access archive for the deposit and dissemination of scientific research documents, whether they are published or not. The documents may come from teaching and research institutions in France or abroad, or from public or private research centers.
L'archive ouverte pluridisciplinaire HAL, est destinée au dépôt et à la diffusion de documents scientifiques de niveau recherche, publiés ou non, émanant des établissements d'enseignement et de recherche français ou étrangers, des laboratoires publics ou privés. 


\title{
Localization of Sources of ELF/VLF Hiss Observed in the Magnetosphere: Three-Dimensional Ray Tracing
}

\author{
L. CaIró \\ Physique Mathématique Modélisation et Simulation, CNRS, Orleans, France \\ F. LEFEUVRE \\ Laboratoire de Physique et Chimie de IEnvironnement, CNRS, Orleans, France
}

\begin{abstract}
Numerical simulation based upon three-dimensional ray-tracing from a single source point located in the equatorial region is used to explain the wave distribution functions (WDF) found for ELF/VLF hiss detected on the GEOS satellite. Emissions at very oblique $\mathbf{k}$ vectors and in all azimuthal directions are shown to produce the GEOS-1 multipeaked WDFs at the longitudes and $L$ values close to the longitudes and $L$ values of a chosen single source point. Inverse ray-tracings, started with the parameters of the WDF's observed emissions, are used to locate the sources.
\end{abstract}

\section{INTRODUCTION}

Wave distribution function (WDF) analyses [Storey and Lefeuvre, 1979, 1980; Lefeuvre and Delannoy, 1979] have considerably modified our knowledge of electromagnetic noises such as VLF hiss [Lefeuvre et al., 1981, 1983; L. R. O. Storey et al., unpublished manuscript, 1986]. Statistics made on GEOS-1 data [Parrot and Lefeuvre, 1986] show that in $\sim 42 \%$ of the cases, the WDFs exhibit two fairly sharp peaks representing two discrete sets of waves. In the other cases, the WDFs are single-peaked indicating generally a wide spreading of wave normal directions. These observations raise two important questions:

1. Do the waves that correspond to a given WDF come from the same source region or not?

2. If yes, can the source be determined, and the propagation characteristics of the waves in that region be obtained from an inverse ray-tracing started from the wave-normal directions given by the WDF analysis?

A preliminary answer to these questions has been obtained by making use of two-dimensional ray-tracing techniques [ $L e-$ feuvre and Helliwell, 1985] (hereinafter referred to as the LH paper). It was noted that, at frequencies below half the electron gyrofrequency, the shape of the phase refractive index surface for whistler mode waves is such that two or more wave-normal directions may exist for a given ray direction. As a result, there are generally several distinct ray paths by which waves can propagate from one point to another in the magnetosphere. Then, if waves are emitted in all permitted wavenormal directions, they may be observed as a double-peaked WDF on a satellite. As a matter of fact, direct ray-tracings conducted from an hypothetical point source located at the equator show that double-peaked WDFs are seen only at $L$ values close to the $L$ value of the source. Inverse ray-tracings were performed from GEOS- 1 data. The best example concerned the September 25 hiss event recorded, outside the plasmasphere, at $L \simeq 6.4$ and at magnetic latitude $\mathrm{LAT}=29^{\circ}$. The source region was located at approximately the same $L$ value $(6.3 \pm 0.2)$ on the opposite side of the magnetic equator $\left(\mathrm{LAT}=-15^{\circ} \pm 5^{\circ}\right.$ ). The initial wave-normals were all very oblique.

Copyright 1986 by the American Geophysical Union.

Paper number 5A8779.

$0148-0227 / 86 / 005 A-8779 \$ 05.00$
Now, two main features of the LH paper have been criticized: first, the hypothesis of an isotropic source (emissions at all permitted wave-normal directions) in the direct study; second, the use of a two-dimensional ray-tracing program in the direct as well as in the inverse study. The present paper, based on the use of a three-dimensional ray-tracing technique, deals with the second point only. As far as the first point is concerned, it is worth mentioning that the general belief, that the instability theory desctibed by Kennel and Petscheck [1966], Etcheto et al. [1973], Church and Thorne [1983] generates longitudinal waves only, is not as strongly founded as it may be thought. The analysis performed on the GEOS data, using a suitable method shows that in the equatorial regions, where it is expected to find the sources, the wave energy is uniformly distributed in wave-normals, the whistler mode resonance angles excluded [Parrot and Lefeuvre, 1986]. Moreover, the calculation of the growth rates with the particle distribution seen on GEOS [Cornilleau-Wehrlin et al., 1985] and with oblique waves [Thorne and Summers, 1986] is just in progress.

The two-dimensional approximation used in the $\mathrm{LH}$ paper was not completely unrealistic, and experience shows that three-dimensional ray-tracing programs are often operated as two dimensional [Cerisier, 1970; Rauch and Roux, 1982]. However, as will be demonstrated in the present paper, the two-dimensional approximation only applies to waves with wave-normals along $B_{0}$ or slightly oblique. Since the WDFs on VLF hiss often peak at very oblique directions, it is highly desirable to study them with the help of a three-dimensional ray-tracing technique.

Theories about three-dimensional ray-tracing have been developed [Cerisier, 1970] and applied to propagation through the ionosphere. They will not be recalled here. In contrast, we have chosen to use the Cerisier program as a tool, first to simulate data and second to perform inverse ray-tracings. As in the $\mathrm{LH}$ paper, in the case studied here a dipole model will be used for the field and a diffusive equilibrium model for the plasma.

The plan of the paper is as follows. The main characteristics of computed ray paths of waves started at the equator are presented in section 2 . In section 3 , the variation of the wavenormal directions along these ray paths is examined. In section 4, data similar to those measured by GEOS and ISEE are obtained assuming an isotropic source, at $L=6.8, \mathrm{LAT}=0^{\circ}$, 
and a satellite crossing the $L$ shells at a constant magnetic latitude value ( $\mathrm{LAT}=20^{\circ}$ ). The location of a source region by inverse ray-tracing is carried out in section 5 for the September 25 hiss event discussed in the LH paper. Finally, section 6 offers some provisional conclusions.

\section{Computed Ray Paths From the Equator}

\subsection{Hypothesis}

The ray-tracing program used here is three dimensional. It is the same as the one developed by Cerisier $[1967,1970]$. The ray paths are computed by a modified version of Haselgrove's ray-tracing equations that is more suitable than the usual one for waves propagating near a resonance. The magnetic field is obtained from the centered dipole model. The refractive index is calculated with the approximation that consists of treating the medium as a cold collision-less plasma composed of electrons and several types of positive ions. The density model is the same as the one defined by Cerisier [1970]. It is derived from the isothermal theory of diffusive equilibrium of electrons and positive ions [Angerami and Thomas, 1964]. Its variation across the lines of force is in $r^{-2.7}$ (at $r \simeq 4$ ), where $r$ is the geocentric distance. This value differs slightly from the variation in $r^{-3}$ given by the Smith [1961] gyrofrequency model. Longitudinal density variations and field gradients are not considered, and ionization ducts are ignored.

The spatial location of the wave is described by its geomagnetic latitude LAT, its geomagnetic longitude LONG, and the magnetic $L$ value. The propagation vector $\mathbf{k}$ is calculated, at each point of space, in a local Cartesian coordinate system $O_{x y z}$ (Figure 1), in which the $O_{z}$ axis is parallel to the earth's magnetic field $\mathbf{B}_{0}$ at the point of observation, the $O_{x}$ axis is in the local magnetic meridian plane and points toward the earth, and the $O_{y}$ axis is oriented westward. In this system, the $\mathbf{k}$ vector is characterized by the polar angle $\theta$ that the wave vector $\mathbf{k}$ makes with the ambient magnetic field, and by the teimuthal angle $\phi$, the origin of which is the $O_{x}$ axis. The $\mathbf{k}$ vector, for which $\phi=0$, is oriented toward lower $L$ shells, whereas the one with $\phi=\pi$ is oriented toward higher $L$ shells. We denote by $\theta_{0}$ and $\phi_{0}$ the $\theta$ and $\phi$ values at the starting point of the ray-tracing.

To avoid confusion, the reader should note that the $\phi$ angle does not measure the deviation of the ray path from a given meridian plane, or to be more explicit, that $\phi=\pi / 2$ or $\phi=3 \pi / 2$ does not mean that the wave propagates perpendicular to the meridian plane.

A parameter that it is important to calculate at each step of the ray-tracing is the normalized frequency $\Lambda=f / f_{c e}$, with $f$ the wave frequency and $f_{c e}$ the local electron gyrofrequency. From the $\Lambda$ value are estimated, at any point of the ray, the values of the whistler mode resonance angle $\theta_{r}$ and of the Gendrin [1961] angle $\theta_{g}$ at which $d(n \cos \theta) / d \theta=0$. In the approximation where the plasma frequency $f_{p e}$ is much greater than the electron gyrofrequency (here $f_{p e} / f_{c e}>3$ ), one has $\cos \theta_{r}=\Lambda, \cos \theta_{g}=2 \Lambda$.

All calculations we present here are done starting the raytracing at the magnetic equator ( $\mathrm{LAT}=0^{\circ}$ ) and at an $L$ value high enough ( $L=6.8$ ) to justify our neglecting the plasmapause (at least in most cases). The values of the plasma parameters and of the magnetic field at the starting point are those currently measured by GEOS-1: (1) electron gyrofrequency at the ground $f_{H o}=943 \mathrm{kHz}$, magnetic field dipolar; (2) electron concentration at the starting point: $3.2 \mathrm{~cm}^{-3} ;(3)$ ionic composition $90 \%$ of $\mathrm{H}^{+}, 10 \%$ of $\mathrm{He}^{+}$.

These parameters correspond for the starting point to an

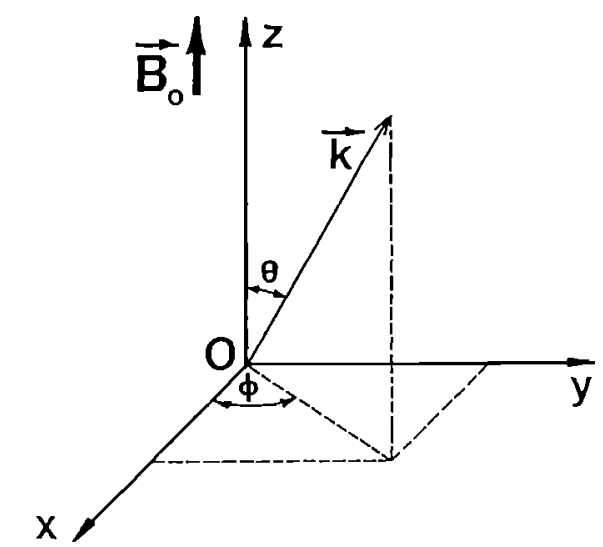

Fig. 1. Coordinate system used in the definition of the wave distribution function.

electron gyrofrequency $f_{c e}$ of $3 \mathrm{kHz}$, a plasma frequency $f_{p e}$ of $16 \mathrm{kHz}$, and a lower hybrid resonance $f_{\mathrm{LHR}}$ of $60 \mathrm{~Hz}$.

Ray-tracings have been performed at different wave frequencies, but for the sake of consistency in the figures the results will only be given for $f=300 \mathrm{~Hz}(\Lambda=0.1$ at the starting point). The conclusions which will be drawn are valid for all waves emitted at $\Lambda \leq 0.5$. Slight differences will be examined at each step.

\subsection{Ray Paths}

Although ray-paths in the magnetic meridian plane have been presented and discussed by several authors (for instance: Cerisier [1970]; Burtis [1973]), we have found it useful to give some extra examples here in order to introduce a few points that we shall develop later. They are displayed in an $L$ versus LAT graph in Figure $2 a$. Two families of rays are distinguishable; one corresponds to waves emitted at $\phi_{0}=\pi$ (full lines), the other to waves emitted at $\phi_{0}=0^{\circ}$ (dotted lines). They are organized according to their $\theta_{0}$ values at the point of emission.

The rays corresponding to the waves emitted at $\phi_{0}=\pi$ (full lines) propagate toward the smaller $L$ values whenever the $\theta_{0}$ value is larger than the local $\theta_{g}$ angle (here $\theta_{g}=76^{\circ}$ ), whereas they are directed first towards higher $L$ values then toward lower $L$ values when the $\theta_{0}$ value becomes smaller than the local $\theta_{g}$ angle. The behavior of the rays corresponding to the waves emitted at $\phi_{0}=0$ (dotted lines) is made more complicated by the existence of a local critical angle $\theta_{c}$ (here $\theta_{c} \simeq$ $50^{\circ}$ ) which depends on the one hand, on the wave and plasma parameters; and on the other hand, on the field gradients [Cerisier, 1970]. The waves started at $\theta_{0}<\theta_{c}$ travel first towards the lower $L$ values then toward the higher. For $\theta$ values included in the interval $\left(\theta_{c}, \theta_{g}\right)$, the rays propagate toward the smaller values. Finally, the waves emitted with $\theta_{0}>\theta_{g}$ propagate toward the higher $L$ values. We use the symbolism parentheses or brackets to specify an open or close interval.

The rays for waves emitted in a plane perpendicular to the geomagnetic meridian plane $\left(\phi_{0}=\pi / 2\right.$ or $\left.3 \pi / 2\right)$ are displayed in Figure $2 b$. There is no difference between rays emitted at $\phi_{0}=\pi / 2$ or at $\phi_{0}=3 \pi / 2$. The rays started with $\theta_{0}$ values smaller than $\theta_{g}$ are directed toward the higher $L$ values, while the ray started with $\theta_{0}$ values greater than $\theta_{g}$ are directed toward the smaller $L$ values.

Obviously, the spread of the rays from the point source strongly depends on the wave frequency and on the exact location of the source, but the main characteristics are the same whatever $f$ (provided $\Lambda<0.5$ ) and $L$. The waves ob- 


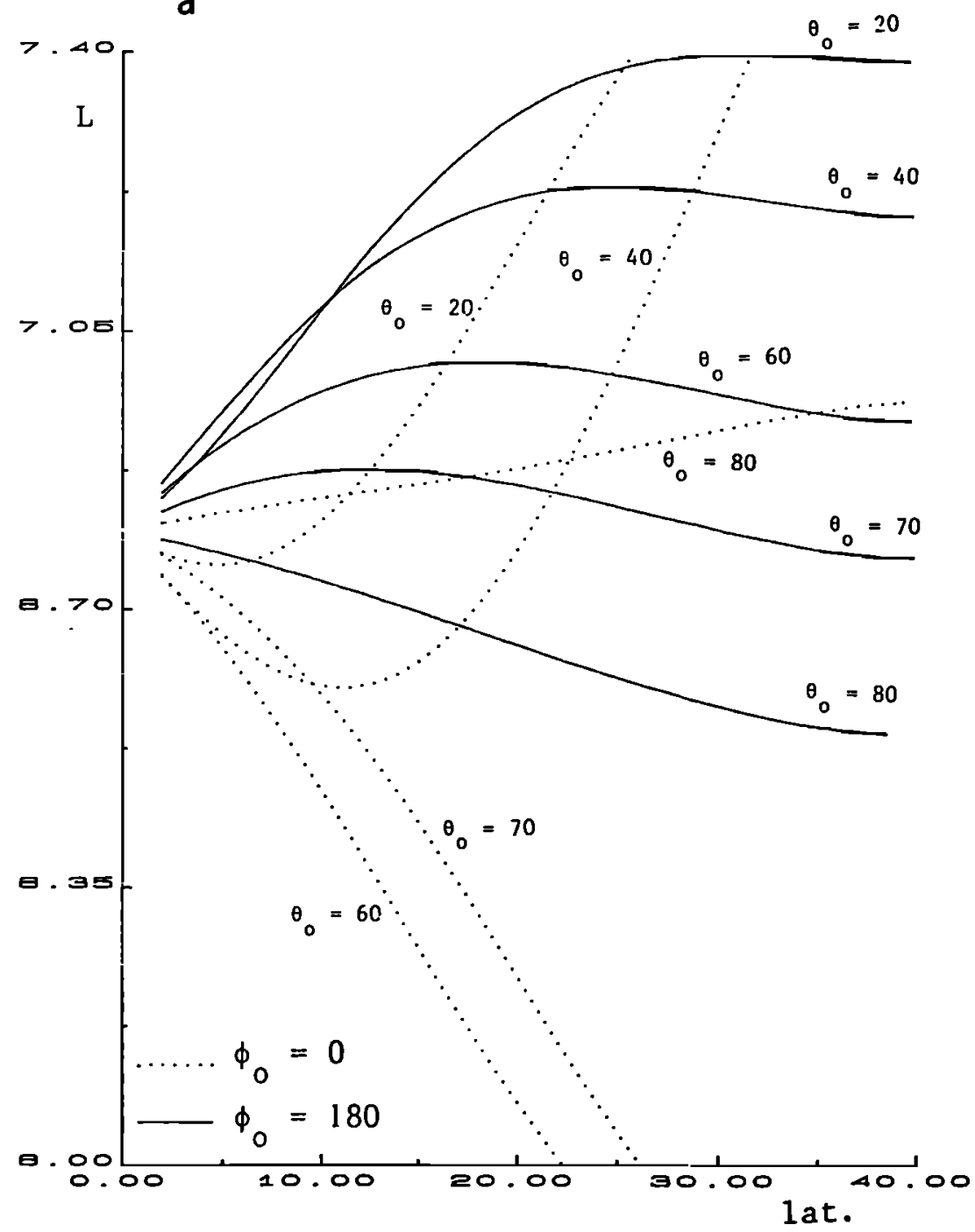

b

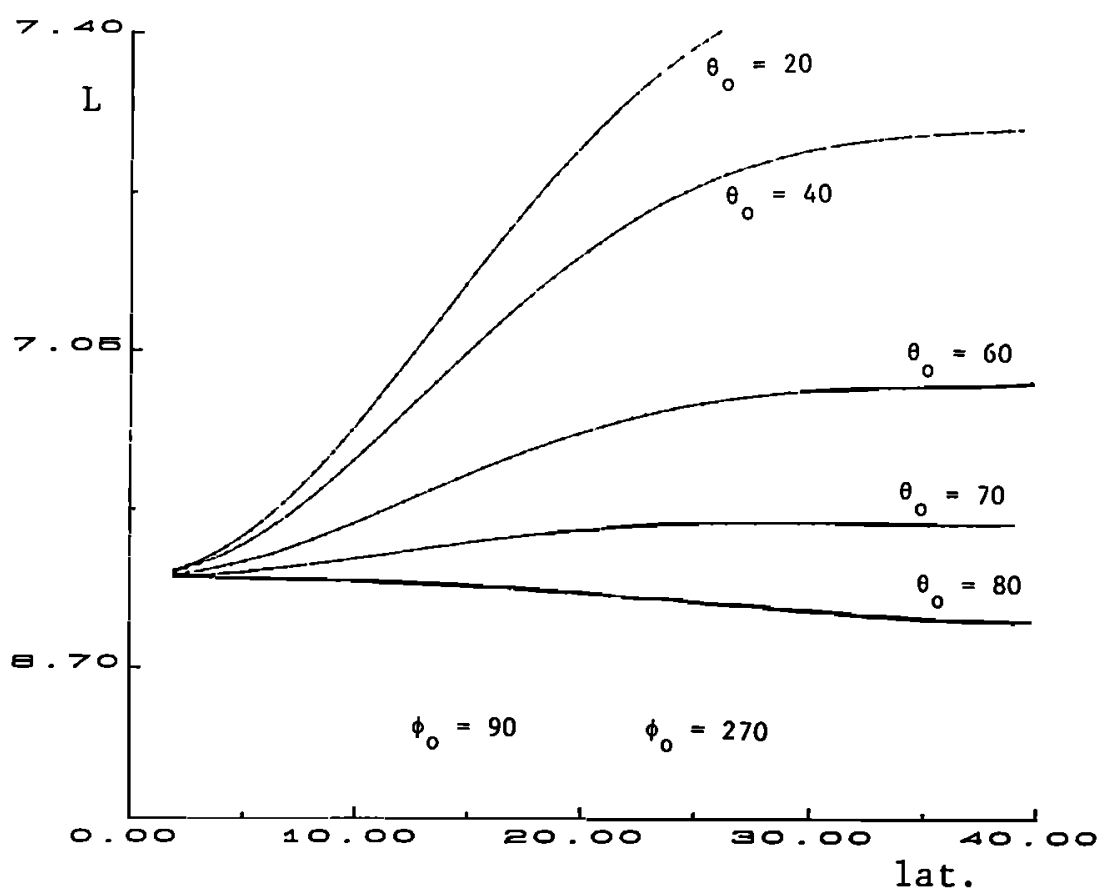

Fig. 2. Ray paths for $300-\mathrm{Hz}$ waves started in (a) the magnetic meridian plane and $(b)$ a plane perpendicular to the magnetic meridian plane. Note that in such $L$ versus LAT plots (here LAT is the magnetic latitude), a ray path along a given line of force is represented by an horizontal line. 
served above the $L$ value of the source correspond generally to waves emitted at small $\theta_{0}$ values but can be accompanied by large $\theta_{0}$ value waves having their $\phi_{0}$ values close to $0^{\circ}$. Below the $L$ value of the source, the waves are generally those emitted at large $\theta_{0}$ values, but they can be crossed by small $\theta_{0}$ value waves having their $\phi_{0}$ values close to $0^{\circ}$.

Now, another point to examine is the probability for the waves emitted at the equator to be returned to their source region in order to maintain the amplification mechanism. Generally, one considers that this probability is null, on the basis of ray-tracings performed assuming $\theta_{0}=0^{\circ}$ and $\phi_{0}=0^{\circ}$ (see, for instance, Thorne et al. [1973]). The case of the plasmaspheric emissions is special since internal reflection from the sharp density gradient at the plasmapause allows the waves to be returned to the source region [Thorne et al., 1979; Church and Thorne, 1983; Huang et al., 1983]. Moreover, taking large $\theta_{0}$ values, and $\phi_{0}$ values in the magnetic meridian plane (Figure 3 ), one easily obtains quasi-cyclic waves. In Figure 3, the wave is started with $\theta_{0}=100^{\circ}$ and $\phi_{0}=0^{\circ}$. It propagates first in the southern hemisphere, toward higher $L$. At the point where the frequency becomes less than the lower hybrid frequency, it experiences a magnetospheric reflection [Kimura, 1966; Lyons and Thorne, 1970]. Next it returns to lower latitudes and crosses the equatorial region, at the $L$ value of the source, its $k$ vector making an angle $\theta=55^{\circ}$ with B. It then propagates in the northern hemisphere till it reaches a second magnetospheric reflection point, which has not been plotted here since the ray returns to the equator at a lower $L$ value. Numerous other examples of waves recycled through the source region are implicit in Figure 8. The mirroring effect is probably less efficient than for ULF waves [Rauch and Roux, 1982], but it clearly exists. However, it may be worth mentioning that in order to maintain the amplification mechanism, it is not sufficient that the waves be returned to their source region: they must be returned with their wave-normals oriented in directions for which the waves are amplified.

\section{Propagation Characteristics}

\section{1. $\theta$ Values}

The $\theta$ values assumed by the $\mathbf{k}$ vector at different points along the ray paths are shown in Figure 4 for four different $\phi_{0}$ values. The source is again located at $L=6.8, \mathrm{LAT}=0^{\circ}$, and the wave frequency is $300 \mathrm{~Hz}$. The $\theta_{0}$ values are obtained by extending the $\theta$ curves till LAT $=0^{\circ}$.

The variations of the $\theta$ values in the geomagnetic meridian plane $\left(\phi_{0}=0^{\circ}\right.$ or $\pi$ ) are quite similar to those described by Thorne and Kennel [1967] and Cerisier [1970]. Waves started at $\phi_{0}=\pi$ attain large wave-normal angles, close to the whistler mode resonance angle, at reasonably low magnetic latitudes. On the other hand, waves started at $\phi_{0}=0^{\circ}$ are split into two families. Large $\theta_{0}$ waves (in fact waves having $\theta_{0}$ values greater than the local $\theta_{\theta}$ ) reach the resonance very quickly. Small $\theta_{0}$ waves $\left(\theta_{0}<\theta_{g}\right)$ have $\theta$ values that first decrease to $0^{\circ}$, where $\phi$ changes from 0 into $\pi$, before migrating toward the large values. Waves started with $\theta_{0}$ values close (but inferior) to $\theta_{g}$ can take such a long time to attain large wave vector angles to the magnetic field that they can reach the ionosphere with quite small $\theta$ values.

The curves obtained for $\phi_{0}=\pi / 4$ and $\pi / 2$ do not exhibit such drastic differences in behavior between waves started at different $\theta_{0}$ values. However, there is a tendency for the small $\theta_{0}$ waves to have $\theta$ values that decrease before going toward the resonance. This indicates that, if most waves reach large wave-normal angles before propagating very far in toward the

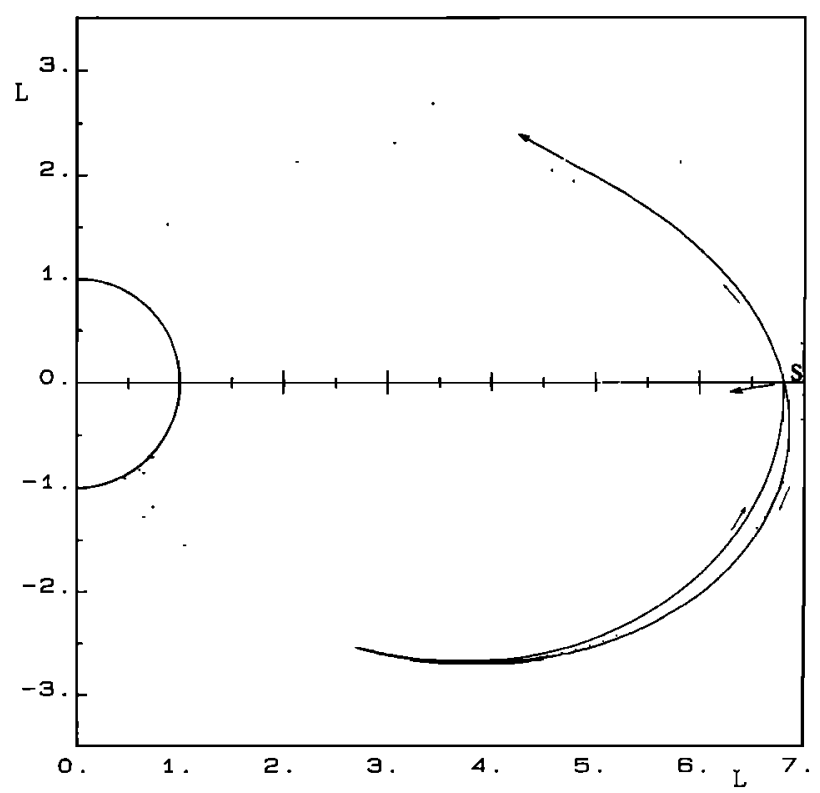

Fig. 3. Ray path, in a magnetic meridian plane, for a wave that returns to the source region. The source is at $S$, and the initial conditions are $\theta_{0}=100^{\circ}, \phi_{0}=0$.

earth, some others, started at $\phi_{0}$ values included in the interval $\left[-45^{\circ},+45^{\circ}\right]$, take a long time to approach the resonance.

Calculations made in the geomagnetic meridian plane for other wave frequencies and source positions [Thorne and Kennel, 1967; Cerisier, 1970] prove that the $\theta$ variations represented in Figure 4 are probably quite general provided that (1) the wave frequency is always smaller than half the electron gyrofrequency at the starting point $(\Lambda \leq 0.5)$; (2) the wave is generated at an $L$ value high enough to allow us to neglect the effects of ionization density gradients on the propagation.

\section{2. $\phi$ Values}

The $\phi$ values taken by the $k$ vectors along the ray paths are shown in Figure 5 for four different $\theta_{0}$ values. The most impressive point is the collimation (more or less rapid, depending on the $\theta_{0}$ values) of all waves into the direction $\phi=180^{\circ}$. Waves leaving the equator propagate towards the earth with $k$ vectors that tend to point toward higher $L$ shells. However, in spite of this tendency, all $\mathbf{k}$ vectors do not reach this orientation. Waves started at $\theta_{0} \geq 50^{\circ}$ take a long time to approach $\phi=\pi$, especially when the $\phi_{0}$ value is around $0^{\circ}$. For $\theta_{0} \simeq \theta_{r}$, the $\phi$ values remain approximately constant all along the ray. Again, there is not much difference in behavior between waves at different frequencies, as one can easily check for frequencies up to a few kHz.

An important consequence of the study summarized in Figure 5 is that WDFs with $\phi$ values around $0^{\circ}$ mainly correspond either to waves observed at the vicinity of the source region or to waves having been generated at large $\theta_{0}$ values. This remark is essential for the understanding of the wave analysis performed on data from GEOS (LH paper) and ISEE [Lefeuvre et al., 1983; Storey et al., 1986].

\subsection{Variations in Longitude}

To have a complete view about the off-meridional propagation, we have plotted in Figure 6, the variations in longitude of the rays. The curves have been drawn for waves started at $\phi_{0}=45^{\circ}, 90^{\circ}$ and $135^{\circ}$, but there is a perfect symmetry with 

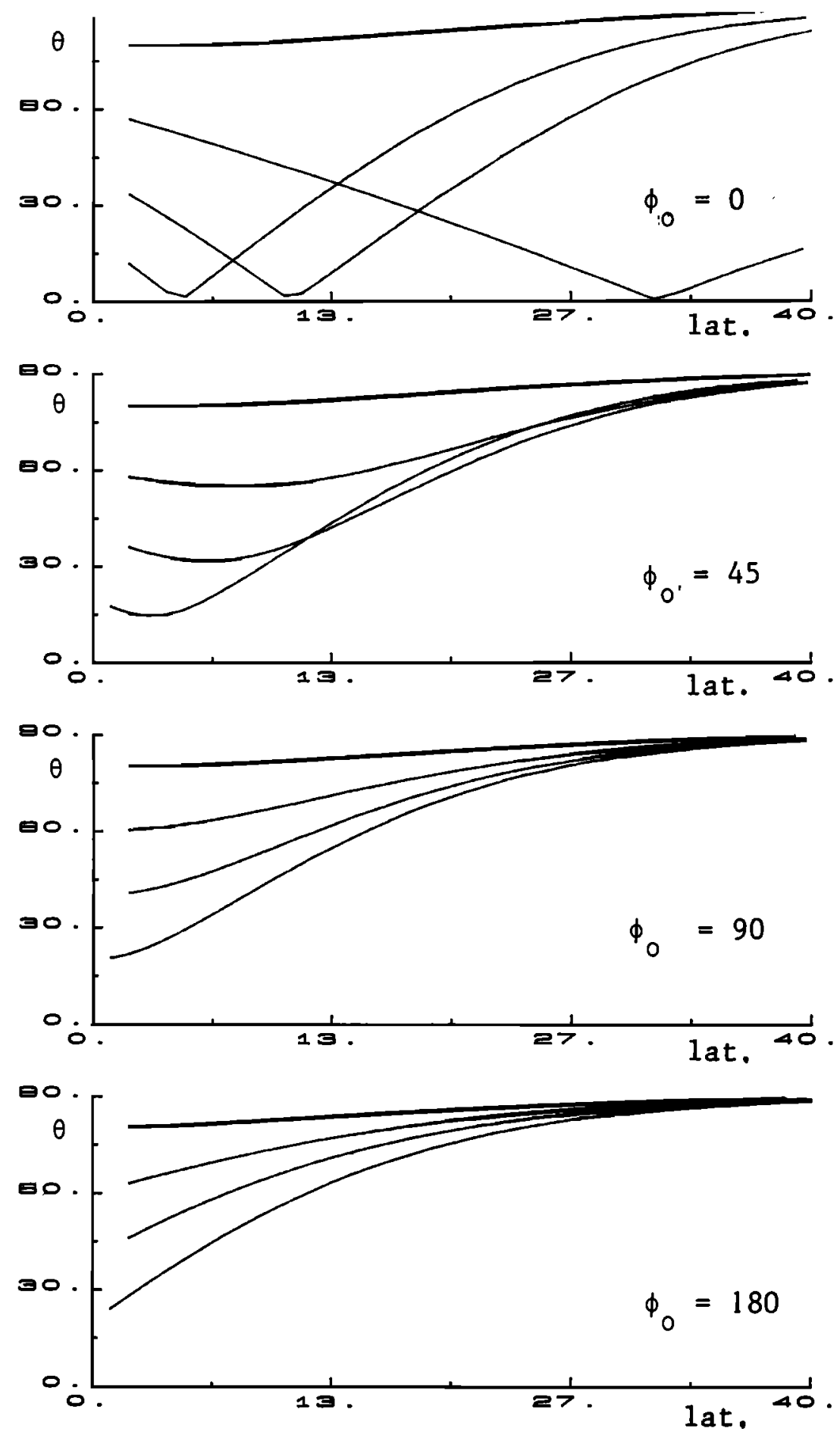

Fig. 4. Variations of the $\theta$ values along the ray path.

the rays started in the other azimuthal quadrants. We note the following three features:

1. The waves started at small $\theta_{0}$ values $\left(\theta_{0}<\theta_{g}\right)$ propagate westward (negative longitudes) while the wave started at large $\theta_{0}$ values $\left(\theta_{0}>\theta_{\theta}\right)$ propagate eastward (positive longitudes).

2. Small $\theta_{0}$ waves move rapidly in longitude at the lowest magnetic latitudes, then converge toward a constant longitude, generally a few degrees off the geomagnetic meridian plane of their starting point, at least if the $\phi_{0}$ value is included in the range $\left[90^{\circ}, 270^{\circ}\right]$.

3. Large $\theta_{0}$ waves move continuously and linearly away from the initial geomagnetic meridian plane.
4. The displacement of the waves in longitude is largest when $\phi_{0}$ is around $0^{\circ}$, then decreases continuously as $\phi_{0}$ increases.

The change in the longitudinal direction pointed out in (1) is due to the change in the ray direction around $\theta_{\theta}$ for waves propagating at $\Lambda \leq 0.5$ [Helliwell, 1965]. As a consequence, rays originating at small $\theta_{0}$ and at $0^{\circ} \leq \phi_{0}<180^{\circ}$ cross rays originated at large $\theta_{0}$ and at $180^{\circ} \leq \phi_{0}<360^{\circ}$ and vice versa. This shows that waves leaving the equator with different $\mathbf{k}$ vectors can cross in longitude as well as they do in $L$ value. From (2) to (4), we also note first, that small $\theta_{0}$ waves started at $\left|\phi_{0}\right| \gtrsim 90^{\circ}$ follow approximately the same sequence of lon- 

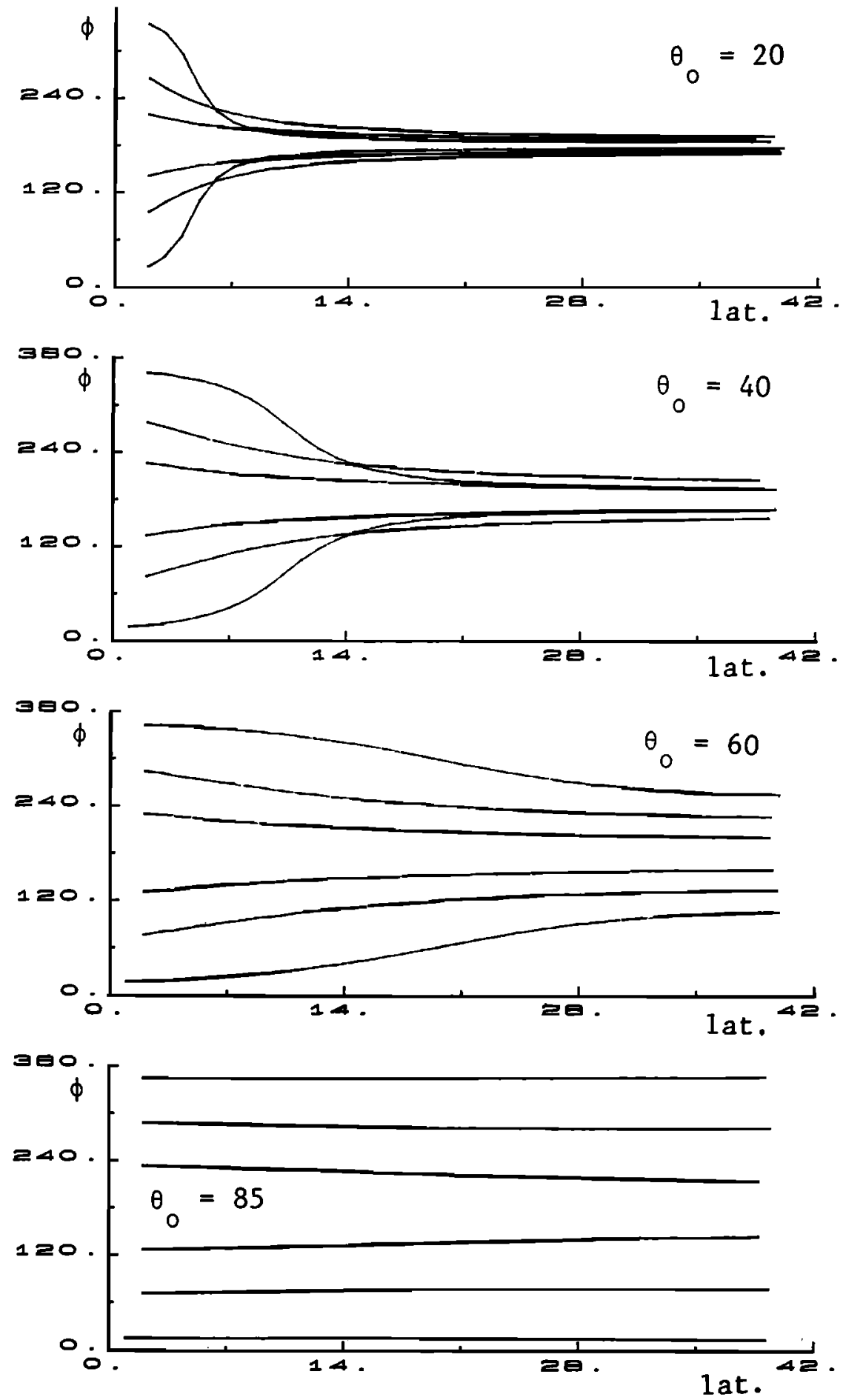

Fig. 5. Variations of the $\phi$ values along the ray path.

gitudes, and second, that their final longitude is slightly different from the longitude of the source.

\section{Simulated Measurements}

\subsection{Conditions for the Simulation}

Now, let us simulate the measurements made on a satellite when the source is an isotropic source (i.e., emitting in all wave normal directions) located at the equator. For this purpose, waves at $300 \mathrm{~Hz}$ are started at $L=6.8, \mathrm{LAT}=0^{\circ}$ LONG $=0^{\circ}$, with wave-normal angles uniformly spaced in $\theta_{0}$ $\left(0 \leq \theta_{0}<\theta_{g}, \Delta \theta_{0}=5^{\circ}\right)$ and $\phi_{0}\left(0 \leqq \phi_{0}<360^{\circ}, \Delta \phi_{0}=5^{\circ}\right)$. The radiation pattern is simulated by tracing the corresponding rays. The observations are supposed to be made on a satellite crossing the $L$ shells at constant geomagnetic latitude (here LAT $=20^{\circ}$ ) and at constant longitude (several passes at different longitudes are considered). A wave is supposed to be detected when its ray crosses the satellite path. The data so simulated are displayed on a LONG versus $L$ plot in Figure 7. Figure $7 a$ refers to the $\theta, \phi$ distributions at LAT $=20^{\circ}$, whereas Figure $7 b$ corresponds to the $\theta, \phi$ distributions at the source for those waves that reach the satellite. Cells of $\Delta$ 

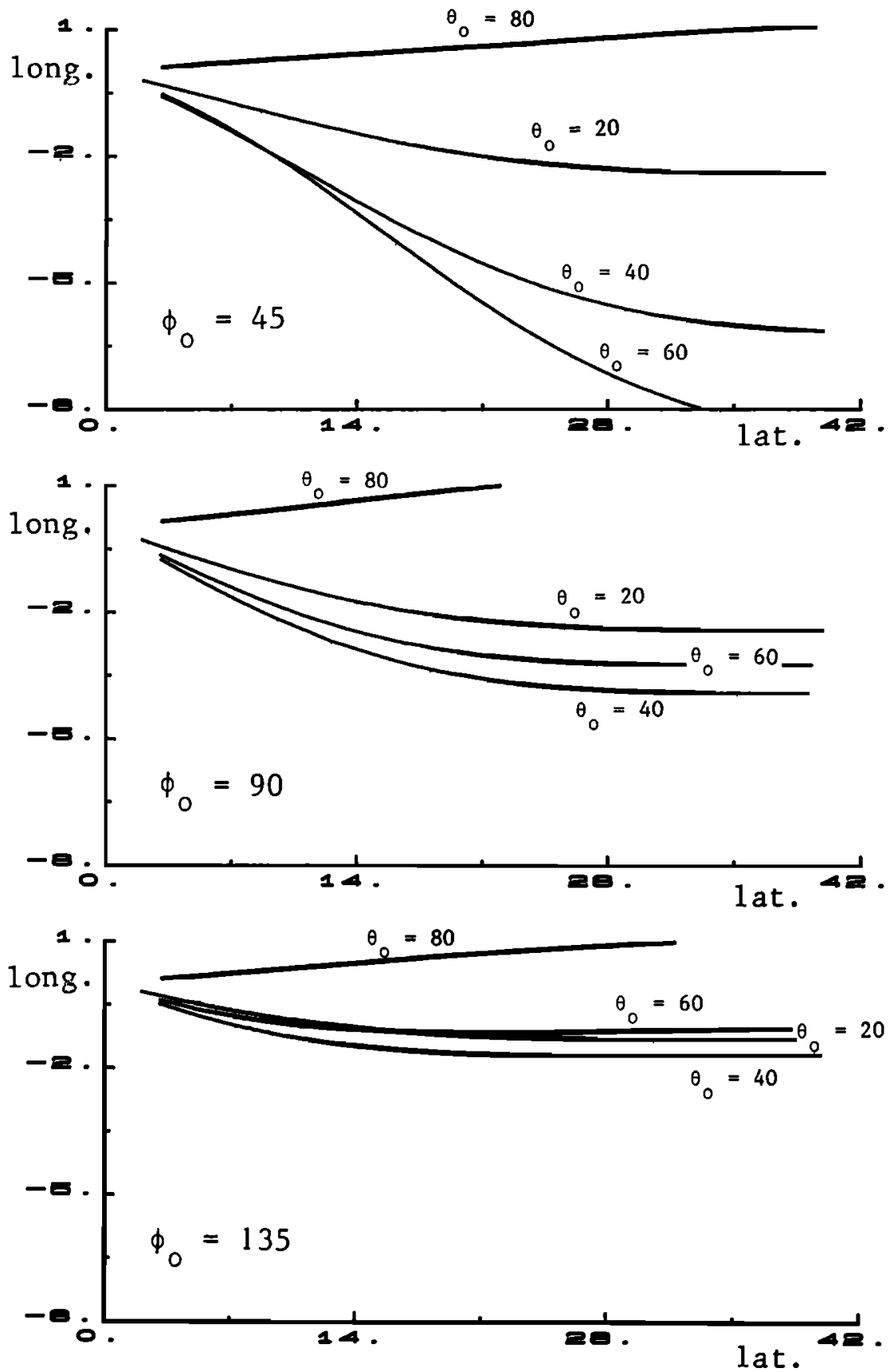

Fig. 6. Variations in longitude along the ray path.

LONG $=0.5^{\circ}$ and $\Delta L=0.3$ are considered. The waves that attain a given cell are characterized by their $\theta$ and $\phi$ values in pseudopolar plots. In such plots, the radial coordinate is proportional to $1-\cos \theta$ while the azimuthal angle is $\phi$. Because of the symmetry in longitude, only negative longitudes are represented.

Three families of waves reaching the satellite are considered. The first consists of waves traveling directly from the source to the satellite. They are termed direct waves and are represented by squares. The second consists of waves having been reflected at the lower hybrid frequency, in the same hemisphere as the satellite. They are termed first reflected waves and are represented by crosses. The third family consists of waves having undergone two such reflections, the second in the opposite hemisphere. They are termed second reflected waves and are represented by triangles.

\subsection{Direct Waves}

$L \geq 6.9$. Let us consider Figure 7a. Far above the source $(L \geq 7.2)$, direct waves are observed only at longitudes close to $0^{\circ}$ (LONG $\geq-3^{\circ}$ ). They have $\theta$ values above $60^{\circ}$ and $\phi$ values at $180^{\circ}$. Figure $7 b$ indicates that they are due to waves started at $\theta_{0} \leq 45^{\circ}$ and $90^{\circ} \leq \phi_{0} \leq 180^{\circ}$. Such behavior is consistent with Figure 2 (section 2.2) and Figure 5 (section 3.2) showing that small $\theta_{0}$ waves are the only ones to propagate toward higher $L$ values and that their $\phi$ values rapidly converge toward $\phi=180^{\circ}$.

Just above the source $(6.9 \leq L<7.2)$, the energy is dispersed throughout all longitudes in the graph. The $\theta$ values vary from $40^{\circ}$ to $90^{\circ}$, whereas the $\phi$ values are included in the interval $\left[130^{\circ}, 180^{\circ}\right]$. Those waves are started at $40^{\circ} \leq \theta_{0}<$ $70^{\circ}$ and $\phi_{0} \simeq 180^{\circ}$ (at the longitude of the source) or $\phi_{0} \rightarrow 90^{\circ}$ (at longitudes different from that of the source). Such behavior 


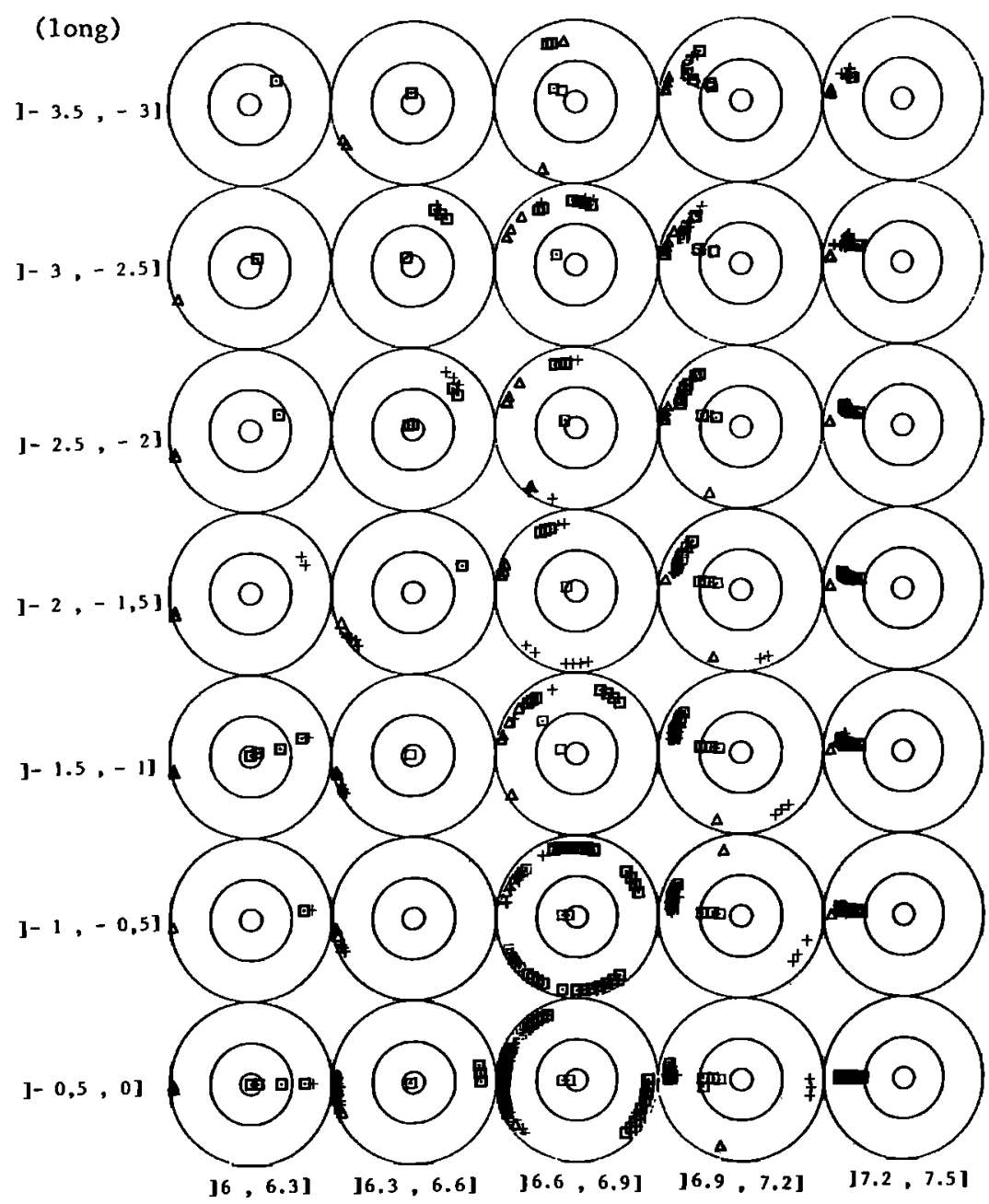

Fig. 7a. Simulation of the $\theta$ and $\phi$ distribution of the waves emitted at the equator at $L=6.8$ and received on a satellite crossing the $L$ shells at a constant magnetic latitude $\left(\mathrm{LAT}=20^{\circ}\right)$; the radii of the circles are proportional to $1-\cos \theta$. Here are represented the $\theta=90^{\circ}, 60^{\circ}$ and $30^{\circ}$ circles. The origin of the angular coordinate $\phi$ is the same as in Figure 9 . The received waves are identified by rectangles (direct waves), crosses (first reflected waves) and triangles (second reflected waves).

is consistent with Figure 6 (section 3.3) showing that the dispersion in longitude increases when $\phi_{0}$ varies from $180^{\circ}$ to $0^{\circ}$.

$L \leq 6.6$. Below the source, waves are seen at all the longitudes shown in the figure. At $L \leq 6.3$, they exhibit $\theta$ values between $0^{\circ}$ and $70^{\circ}$ and $\phi$ values between $0^{\circ}$ and $90^{\circ}$, the value $\phi=0^{\circ}$ being obtained only at LONG $\simeq 0^{\circ}$. As it could be expected from section 3 (Figure $2 a$ ), those waves are generated at moderately large $\theta_{0}$ values $\left(\theta_{0} \sim 65^{\circ}\right)$ for $\phi_{0} \simeq 0^{\circ}$. Just below the source $(6.3<L \leq 6.6)$ the waves are more dispersed in $\theta$ and $\phi$ values $\left(10^{\circ} \leq \theta<85^{\circ} ; 0 \leq \phi \leq 180^{\circ}\right), \phi$ taking values close to $0^{\circ}$ at LONG $\simeq 0^{\circ}$ and to $90^{\circ}$ at LONG $\rightarrow$ $-5^{\circ}$. The corresponding rays are started at $45^{\circ} \leq \theta_{0} \leq 80^{\circ}$ and $0^{\circ} \leq \phi_{0} \leq 20^{\circ}$. Again only small $\phi_{0}$ waves are seen.

$L \sim 6.8$. At the $L$ value of the source, the waves are mainly observed with $\theta$ values around $85^{\circ}$ but a few are seen with $\theta \leq 30^{\circ}$. The $\phi$ values are grouped between $45^{\circ}$ and $180^{\circ}$ except at longitudes close to $0^{\circ}\left(\right.$ LONG $\left.\geq-1^{\circ}\right)$ where $0^{\circ} \leq \phi<360^{\circ}$. The dispersion in $\phi$ values is obviously a consequence of the emission at large $\theta_{0}$ values $\left(\theta_{0} \geq 85^{\circ}\right)$ for which the $\phi$ values remain nearly constant in the course of the propagation (see Figure 5, section 3.2).

\subsection{Reflected Waves}

$L \geq 6.9$. Far above the source $(L \geq 7.2)$, the reflected waves are seen at $\theta \simeq 70^{\circ}, \phi_{0} \simeq 180^{\circ}$. They are due to waves that started at small $\theta_{0}\left(\theta_{0} \leq 60^{\circ}\right)$ with $\phi_{0}$ values between $90^{\circ}$ $\left(\right.$ LONG $\rightarrow-4^{\circ}$ ) and $180^{\circ}$ (LONG $\rightarrow 0^{\circ}$ ). Just above the source $(6.9 \leq L \leq 7.2)$ they are observed at large $\theta$ values and have $\phi$ values widely dispersed over the whole interval $(0,2 \pi)$. They are due either to waves started at $0^{\circ} \leq \theta_{0} \leq 60^{\circ}$, with $\phi_{0}$ values between $90^{\circ}$ and $180^{\circ}$, or, which distinguishes them from the previous case, to large $\theta_{0}$ waves emitted at $\phi_{0} \rightarrow 0^{\circ}$ ( $90^{\circ}$ or $270^{\circ}$ at higher longitudes).

$L \leq 6.6$. Far below the source $(L \leq 6.3)$, the reflected waves have large $\theta$ values, and $\phi$ values around $0^{\circ}$ as well as around $180^{\circ}$ They have practically the same propagation characteristics all along their ray paths. Just below the source $(L \leq 6.6)$ they have large $\theta$ values having $\phi$ values around $180^{\circ}$. They are due to waves emitted at $60^{\circ}<\theta_{0} \leq 90^{\circ}$ with any $\phi_{0}$ value.

$L \simeq 6.8$. Surprisingly, the reflected waves detected at the $L$ values of the source have nearly same propagation characteristics as they have just below the source. The only difference is a wider spread in $\phi$ values around $180^{\circ}$.

\subsection{Global Picture}

At longitudes close to the longitude of the source, going from high to low $L$ values, we find (1) above the $L$ value of the source, direct and reflected waves with large $\theta$ values and $\phi$ values around $180^{\circ} ;(2)$ at the $L$ value of the source, waves 


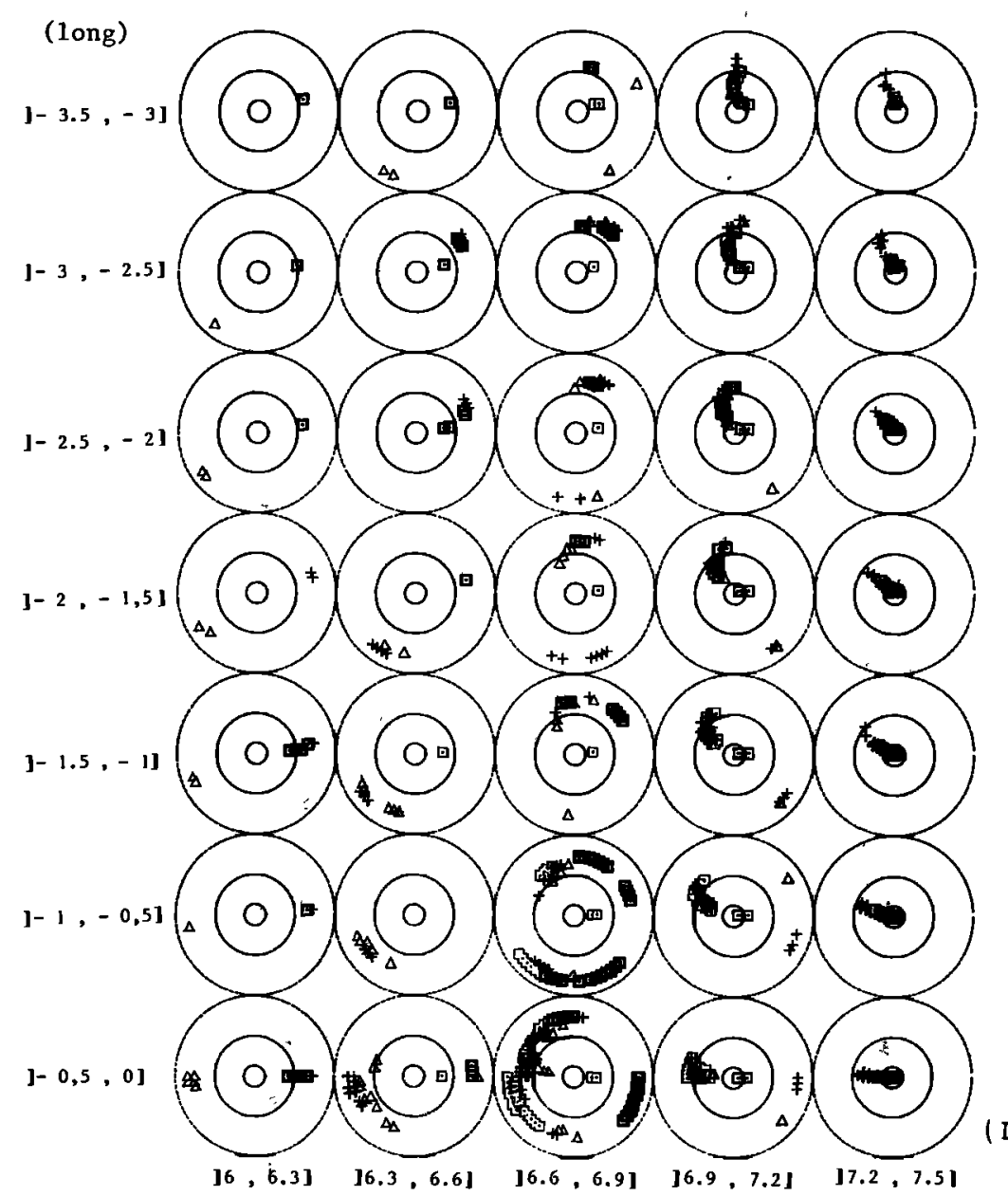

Fig. $7 b$. Corresponding $\theta_{0}$ and $\phi_{0}$ angles for the waves simulated in Figure $7 a$. The notation is the same as in this figure.

widely spread in $\theta$ and $\phi$ values $\left(0^{\circ} \leq \dot{\theta}<85^{\circ} ; 0^{\circ} \leq \phi<360^{\circ}\right)$, the reflected waves having the largest $\theta$ values and being more or less concentrated around $\phi=180^{\circ} ;(3)$ below the $L$ value of the source, waves widely spread in $\theta\left(0^{\circ} \leq \theta<85^{\circ}\right)$ but having $\phi$ values either around $0^{\circ}$ (direct waves plus a few reflected waves) or around $180^{\circ}$ (reflected waves).

As a matter of fact, the distribution in $\phi$ values of the direct waves is very similar to observations made during an equatorial pass of the ISEE satellite [Lefeuvre et al., 1983]. During that pass, the electromagnetic emissions are characterized: at the highest $L$ values $(L \geq 3.6)$, by one-peaked WDFs centered at $\phi \simeq 180^{\circ}$; at medium $L$ values $(3.6<L<2.9)$, by twopeaked WDFs centered at $\phi \simeq 180^{\circ}$ and at $\phi \simeq 0^{\circ}$; at the lowest $L$ values $(\mathrm{L}<2.9$ ), by one-peaked WDFs centered at $\phi \simeq 0^{\circ}$. This seems to indicate that the source is located between $L=3.6$ and $L=2.9$, which is supported by an increase in the wave intensity in this region. The reflected waves probably convey much less energy than the direct waves. This is consistent with our numerical simulations showing that, in each cell of Figure 7, the number of direct waves is much greater than the number of reflected waves.

At larger longitudes, the picture is less clear. Again, there is a greater dispersion in the $\phi$ values at the $L$ value of the source, but it covers the half interval $\left[0^{\circ}, 360^{\circ}\right)$ at the best $\left(90^{\circ}\right.$ to $270^{\circ}$ for negative longitudes). Moreover, at high as well as at low $L$ values, $\phi$ tends towards $90^{\circ}$ (negative longitudes) or $270^{\circ}$ (positive longitudes). In both cases, many more direct waves reach the satellite than reflected waves. This means that the main part of the wave energy is conveyed by direct waves.
As far as the observation of the double-peaked WDF's is concerned, it is cleari that (1) they can be produced by direct waves, without any heed of reflected waves, at least at longitudes and $L$ values close to those of the source, and (2) the probability of having double-peaked WDFs generated by reflected waves only is small but not zero.

\subsection{Reinjection in the Source Region}

For the sake of completeness, the reflected waves observed in the equatorial region, at longitude and $L$ values close to those of the source, have been represented in Figure 8. They generally propagate at $\theta$ values much greater than the $\theta_{0}$ values at the source, and have $\phi$ values included in the same interval $\left[90^{\circ}, 270^{\circ}\right]$ as their $\phi_{0}$ values. Contrariwise, small $\theta$ waves are generated by large $\theta_{0}$ waves started at $\phi_{0} \simeq 0^{\circ}$, in accordance with section 3.1 (Figure 4).

Even if the recycled waves have not exactly the same propagation characteristics as their parent waves, it is clear that a nonnegligible amount of wave energy is returned into the source region by nonducted waves allowing for the maintaining of an amplification mechanism.

\section{Localization of a Source Region}

\subsection{Hypothesis}

If multipeaked WDFs are in fact generated by waves leaving the same source region with different $\mathbf{k}$ vectors, there are no theoretical difficulties in trying to locate such a region from 


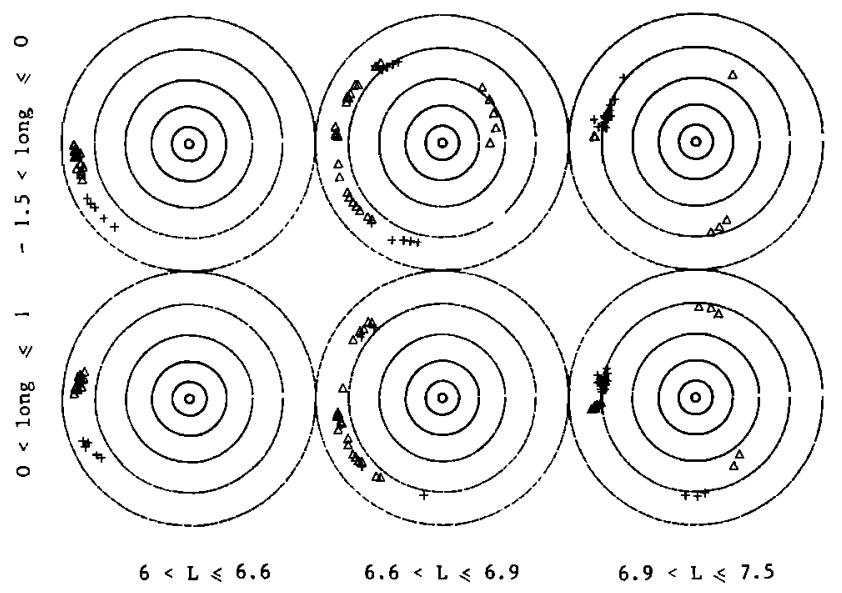

Fig. 8. $\theta$ and $\phi$ distribution of the waves when they have returned to the source region $\left(L=6.8\right.$, magnetic latitude LAT $=0^{\circ}$, geographical longitude LONG $=0^{\circ}$ ). Here the circles represent $\theta=15^{\circ}, 30^{\circ}$, $45^{\circ}, 60^{\circ}, 75^{\circ}$ and $90^{\circ}$.

ray-tracings started at the point of observation with $\mathbf{k}$ vectors opposite to the $\mathbf{k}$ vectors of the maxima of the WDF (see the LH paper). In practice there are difficulties related to the possible extension of the source region in space and to the modeling of the medium. One way of approaching a solution would be to record the same emission over long distances along the satellite orbit, and to perform inverse ray-tracings from different points of space, in order to use some kind of triangulation technique. Now, when the satellite moves in $L$ values over intervals large enough $(\Delta L \gtrsim 0.5)$ to satisfy the requirements for the precision of the ray-tracing, it generally encounters different media. For instance, it has been shown that $66 \%$ of the GEOS VLF hiss observed outside the plasmasphere are associated with enhancements in the electron density of the order of $30 \%$ or more [Parrot and Lefeuvre, 1986]. However, the results obtained by ray-tracings are approximate only since longitudinal density variations and field gradient are not included in our model.

As a matter of fact, the conditions for an inverse ray-tracing are so strict (stationarity in time of the signal, high signal-tonoise ratio, multipeaked WDF, electron density measurements consistent with a diffusive equilibrium model, point of observation far from the plasmapause and from any irregularity in electron density, ambient magnetic field measurements consistent with a dipolar model), that we had difficulties in finding even one GEOS situation where a trial could be performed. From this unique inverse ray-tracing, we only want to demonstrate first, that rays cross in longitude as well as in $L$ value. In other words, that rays observed at a given point, located at sufficiently high geomagnetic latitude, seem to emerge from the same longitudinal region, and second, that even if the sources are still located ambiguously, the estimate of the distribution in wave normals within such sources may be unambiguous.

\subsection{GEOS-1 Data}

The GEOS data that we have finally considered refer to the September 25, 1977, hiss event already studied in the $\mathbf{L H}$ paper. The observation were made outside the plasmasphere around 1315:44 UT at $L=6.40, \mathrm{LAT}=29.2^{\circ}, \mathrm{MLT}=1400$ hours. For a description of the wave experiment, see $S-300$ Experimenters [1979].

A WDF analysis has been performed from the measurements of the three magnetic components, at two different fre- quencies $(825 \mathrm{~Hz}$ and $923 \mathrm{~Hz})$ and at two different times $44 \mathrm{~s}$ apart. The quantity determined is $F(f, \cos \theta, \phi)+F(f,-\cos \theta$, $\phi+\pi)$ which, because of its symmetry is only graphed for one hemisphere (here $\theta \leq \pi / 2$ ). The four solutions are displayed in Figure 9. On each graph, the scale of $F(f, \cos \theta, \phi)$ is linear and runs from 0 to 10 . The outer circle corresponds to $\theta=90^{\circ}$ while the inner circle indicates the position of the resonance cone for the whistler mode $\left(\cos \theta_{r}=f / f_{c e}\right)$. Slight differences between the solutions, especially in the $\phi$ values are easily explained by the errors in the data. (Note that the $O_{y}$ axis points westward here and eastward in the LH paper.) As a first approximation, we can consider that the wave energy is conveyed by two wave packets. The more intense has $\theta$ and $\phi$ values such that $70^{\circ} \leq \theta \leq 80^{\circ}, 180^{\circ} \leq \phi \leq 230^{\circ}$. The other is characterized by $15^{\circ} \leq \theta \leq 35^{\circ},-60^{\circ} \leq \phi \leq 40^{\circ}$. Although the analysis of the magnetic plus the electric data has not allowed us to remove the ambiguity on the sign of the wavenormal direction, we assume that, as for the June 26, 1977, hiss event reported in the LH paper, the two wave packets have a component of velocity parallel to the field $\mathbf{B}_{0}$ of the same sign.

Electron densities are measured on GEOS both by a relaxation sounder (S 301) and by a mutual impedance experiment (S 304). An on-board magnetometer (S 331) provides the measurements of the earth magnetic field vector. At the point of observation, the plasma frequency is $29.2 \mathrm{kHz}$ and the electron gyrofrequency is $7.173 \mathrm{kHz}$. Comparisons between the measurements made over several UT intervals, assuming that the observations are stationary in time, enable us to have an idea about the main characteristics of the propagation medium. The plasmapause is located at an $L$ value estimated between 5.5 and 6 . The electron density (see Table 1) has a mean variation in $r^{-3}$, and the earth's magnetic field varies as $r^{-2}$. Although both values do not fit exactly the parameters of the model given in section 2, the results of our inverse raytracing should not be too much influenced as the ray propagates at nearly constant $L$ values.

\subsection{Inverse Ray-Tracing}

Inverse ray-tracings have been performed from the observation point. The most intense wave packet has been simulated starting the rays at $\theta=78^{\circ} \pm n \Delta \theta, \phi=180^{\circ} \pm m \Delta \phi$, and the other packet starting the rays at $\theta=22^{\circ} \pm m \Delta \theta$, $\phi=13^{\circ} \pm m \Delta \theta$, with $\Delta \theta=\Delta \phi=1^{\circ}, n$ and $m$ each run from 0 to 5 . The left diagram in each panel of Figure 10 represents the $L$ and LONG values at which each ray crosses planes of constant geomagnetic latitude. The crossing points of the rays form distinct clusters. The bottom one at LAT $=25^{\circ}(\mathrm{A})$, corresponds to the first wave packet $\left(\theta \simeq 78^{\circ}, \phi \simeq 180^{\circ}\right)$ and the top one (B) to the second $\left(\theta \simeq 22^{\circ}, \phi=13^{\circ}\right)$. In these clusters, the lines represent the dispersion in $\theta$ values while the columns correspond to the dispersion in $\phi$ values. Between $-10^{\circ}$ and

TABLE 1. Field and Plasma Parameters as Measured by GEOS

\begin{tabular}{lccc}
\hline & \multicolumn{3}{c}{ Value } \\
\cline { 2 - 4 } Parameter & $1200 \mathrm{UT}$ & $1300 \mathrm{UT}$ & $1315 \mathrm{UT}$ \\
\hline LAT & $28.2^{\circ}$ & $29.3^{\circ}$ & $29.4^{\circ}$ \\
LONG & $4.6^{\circ}$ & $2.2^{\circ}$ & $3.3^{\circ}$ \\
Distance, $\mathrm{R}_{\mathrm{T}}$ & 6.10 & 5.18 & 4.92 \\
$F_{\text {ce }}, \mathrm{kHz}$ & 4.93 & 6.68 & 7.17 \\
$F_{\text {pe }}, \mathrm{kHz}$ & 21.44 & 30.49 & 29.20 \\
\hline
\end{tabular}

LAT is the magnetic latitude and LONG is the geographical longitude. 

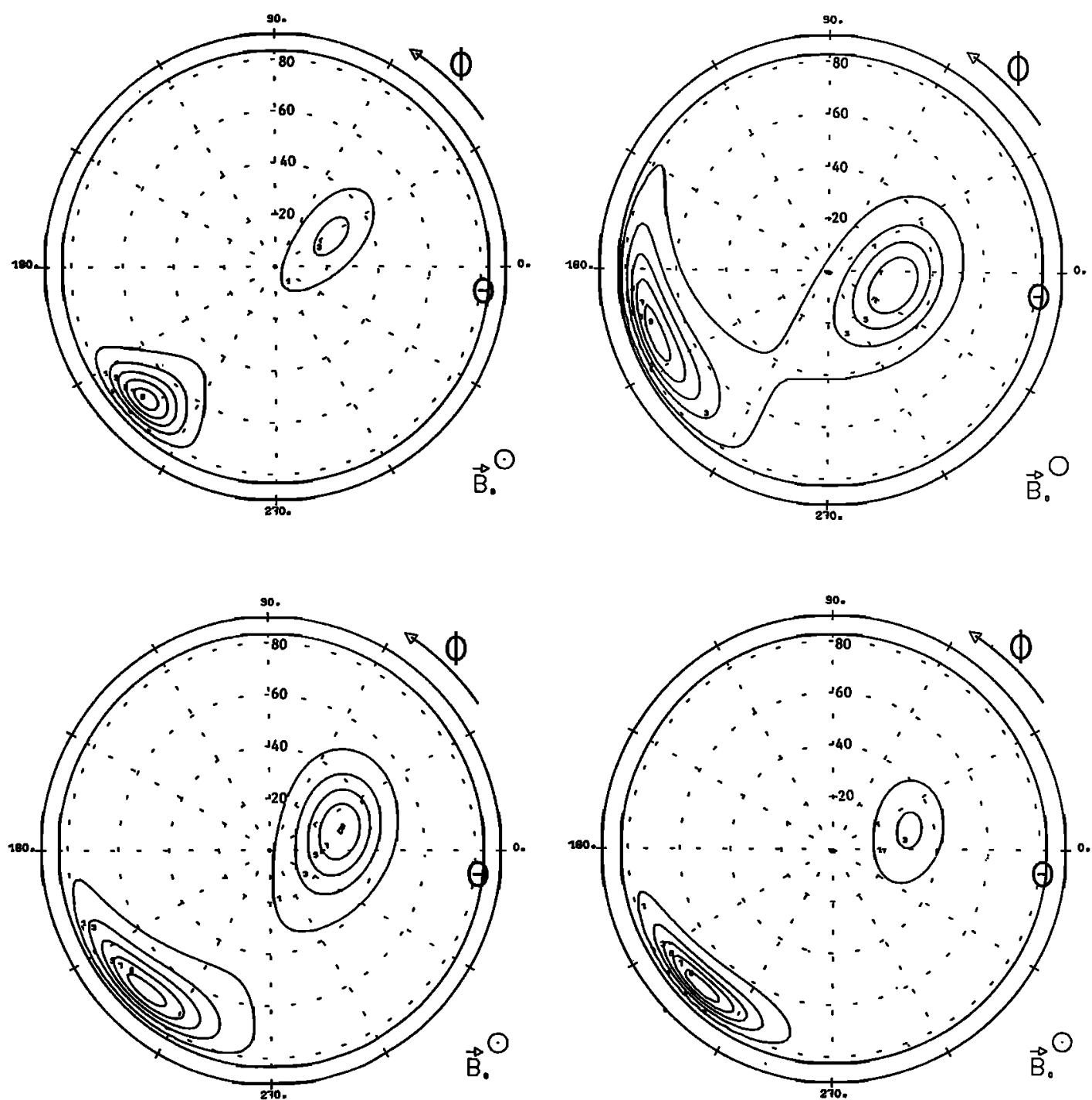

Fig. 9. Contours of the WDFs obtained for the September 25, 1977, hiss event observed on GEOS-1. The analysis is made for two different time intervais (left and right panels) $44 \mathrm{~s}$ apart, and at two different frequencies (825 $\mathrm{Hz}$, top panel; $923 \mathrm{~Hz}$, bottom panel). The scale of the WDF is linear and runs from 0 to 10 . The outer circle corresponds to $\theta=90^{\circ}$ while the inner circle indicates the position of the resonance cone for the whistler mode.

$-30^{\circ}$, the two clusters merge and farther on (not shown here) they diverge. The polar plots, at the right side of Figure 10, indicate the $\theta$ and $\phi$ values of the different waves at fixed magnetic latitudes. They are grouped into two narrow clusters which practically merge at LAT $=-30^{\circ}$.

A first interpretation of this inverse ray-tracing is that the rays reaching the GEOS satellite on September 25,1977 , emerge from a source region located at $-10^{\circ} \leq \mathrm{LAT} \leq-30^{\circ}$ on the opposite side of the equator at approximately the same $L$ and LONG values. The wave energy in the source region is conveyed by waves having large $\theta$ values and $\phi \simeq 0^{\circ}$ Accordingly to the imperfection in the modelling of the medium, we know that this picture is far from being the real picture. But it is clear that double-peaked WDFs can be explained by waves propagated from isotropic sources localized in space.

Now, looking at Figure 10 in the opposite direction (from $\mathrm{LAT}=-30^{\circ}$ to $\mathrm{LAT}=25^{\circ}$ ), let us propose another interpretation, which is not in complete contradiction with the first one. Waves originated for instance at the equator and having suffered a reflection at a strong density gradient (plasmapause or irregularities in electronic density) or/and at the lower hybrid resonance may reach the domain represented at the bottom of Figure $10\left(\mathrm{LAT}=-30^{\circ}\right)$ with wave-normals slightly dispersed, but characterized by large $\theta$ values and $\phi$ values around $0^{\circ}$. If so, due to the propagation effects, the wave energy can be split into two separate packets. Smaller $\theta$ waves have their $\theta$ values first decreasing, then increasing when all the $\phi$ values have attained $180^{\circ}$. Larger $\theta$ waves remain at $\phi \simeq 0^{\circ}$, but then have $\theta$ values slowly decreasing to $0^{\circ}$. They recombine into a single packet at $\mathrm{LAT}>25^{\circ}$, after having followed different ray paths.

Both interpretations suppose that the amplification mechanism be effective on large $\theta$ waves as well as on small $\theta$ waves, or at least, that the Landau damping be negligible even at very oblique propagation.

\section{Conclusion}

The propagation of ELF and VLF emissions below half the electron gyrofrequency has been studied numerically using the Cerisier [1970] three dimensional ray-tracing program. The ambient field is modeled as an earth-centered dipole. The density model is derived from the isothermal theory of diffusive 
L
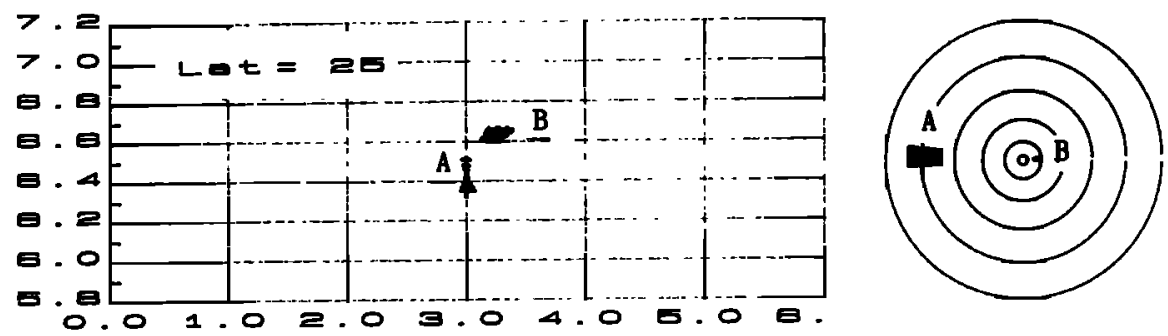

long

L
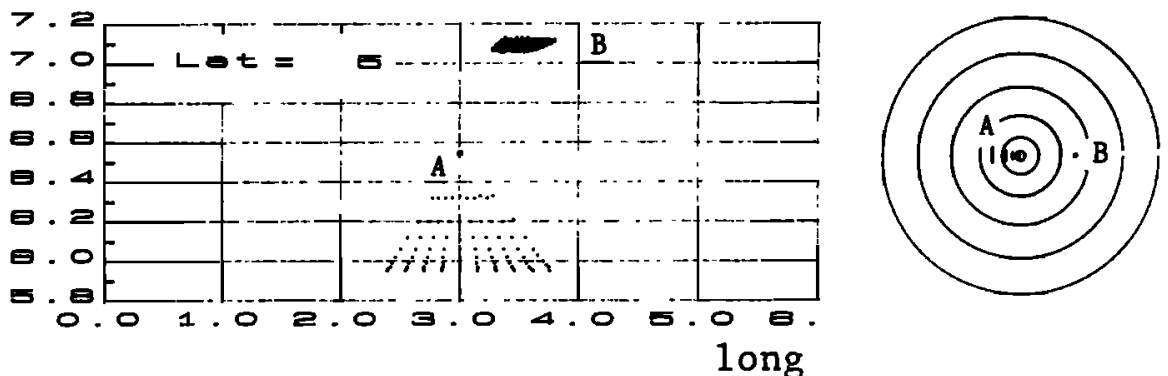

L
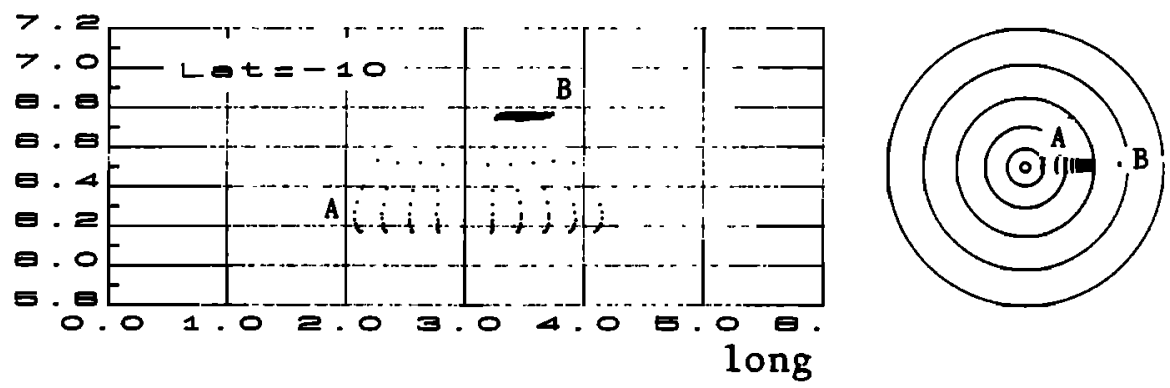

L
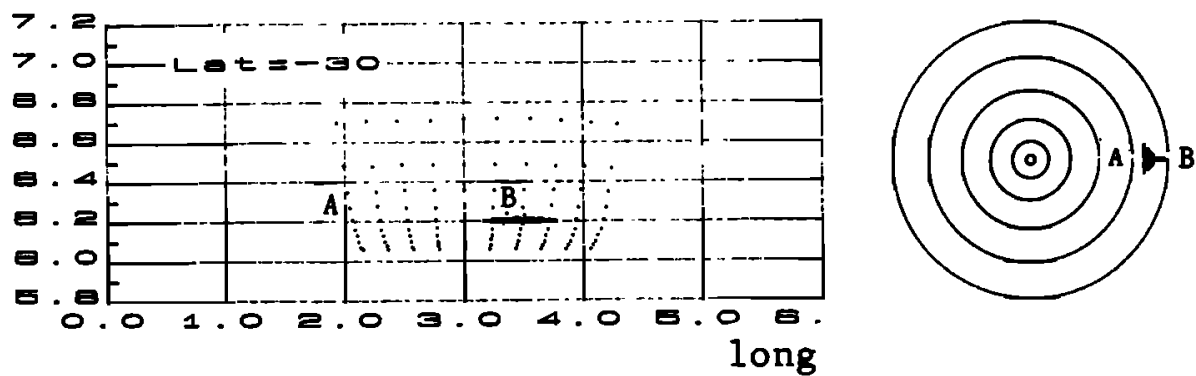

Fig. 10. Localization by inverse tracing of the source for the September 25,1977 , hiss event observed on GEOS-1 at $L=6.4$, magnetic latitude, $L A T=+29.2^{\circ}$, geographical longitude, LONG $=3^{\circ}$. On the left, the dots represent the position in LAT and LONG of the rays started from the observation point with the $\theta$ and $\phi$ values of the peaks of the WDFs (see text). On the right are represented the $\theta, \phi$ distributions of the corresponding waves.

equilibrium of electrons and positive ions. The study has been made first assuming an isotropic source located at the equator. The main propagation characteristics derived from the ray tracing are as follows.

1. The waves reach large wave normal angles before propagating very far in toward the earth, unless they are started at $\phi_{0}$ values close to $0^{\circ}$.

2. Except for waves normal started with a $\phi_{0}$ value strictly equal to $0^{\circ}$, the $\phi$ values converge toward $180^{\circ}$; the steepness of this convergence is inversely proportional to the $\theta_{0}$ value. Large $\theta_{0}$ waves see their $\phi$ values nearly constant in the course of the propagation, while small $\theta_{0}$ waves have all their $\phi$ values close to $180^{\circ}$ at reasonably low magnetic latitude.

3. The waves emitted westward $\left(0 \leq \phi_{0} \leq 180^{\circ}\right)$ propagate westward in longitude when started at $\theta_{0}$ values smaller than the $\theta_{g}$ angle, whereas they propagate eastward in longitude when started at $\theta_{0}$ values greater than $\theta_{g}$. Obviously, the opposite is true for waves emitted eastward.

4. Direct waves observed far above the $L$ value of the source are generally waves emitted at small $\theta_{0}$ values $\left(\theta_{0}<\right.$ $60^{\circ}$ ). They are seen at all $\theta$ values but their $\phi$ values concentrated around $180^{\circ}$. Direct waves observed far below the $L$ 
value of the source are also waves emitted at small $\theta_{0}$ values $\left(\theta_{0}<60^{\circ}\right)$; however, they are started with $\phi_{0}$ values around $0^{\circ}$. They are seen at all $\theta$ values but have their $\phi$ values concentrated around $0^{\circ}$. At $L$ values close to that of the source, all types of waves can be seen.

5. A nonnegligible amount of wave energy is recycled to the source region by unducted waves generated at medium and large $\theta_{0}$ values.

A simulation of the observations made by a satellite crossing the $L$ shells at constant magnetic latitude has shown that the multipeaked WDFs of the GEOS data can be interpreted by waves started at the equator with different $\theta_{0}$ and $\phi_{0}$ values and reaching the satellite after having followed different ray paths. Although most of the multipeaked WDF $s$ are the result of the crossing of direct waves, there is a possibility of obtaining identical distributions by the crossing of direct waves plus reflected waves and even by the crossing of reflected waves only. The probability of the observation of a multipeaked WDF is highest at $L$ and LONG values close to those of the source.

Inverse ray-tracing has been used to locate the source of a VLF emission observed on GEOS-1 outside the plasmasphere. The parameters of the theoretical medium are adjusted to the electron gyrofrequency and to the plasma frequency measured on GEOS-1. The rays are started at the point of observation with the $\theta$ and $\phi$ values of the peak of the WDF. It is concluded that the waves emerge from a region located on the opposite side of the magnetic equator (LAT $=-20^{\circ}$ ) at approximately the same $L$ and LONG values, the initial wave normal directions being very oblique. As very well illustrated in Figure 10, this region can be interpreted either as a source or as a region where the wave energy splits into two separate groups. Such results confirm the conclusions of the LH paper.

The possibility that the waves could be generated at some prefered angles in some finite-extend source region, and then propagate several equatorial transits before they are observed cannot be ruled out, but seems improbable according to the spread in the wave energy in the course of the propagation.

Acknowledgments. The authors wish to express their sincere thanks to J. C. Cerisier for helpful discussions and remarks, and they especially thank the referees for very useful comments and grammar corrections.

The Editor thanks L. R. O. Storey and another referee for their asistance in evaluating this paper.

\section{REFERENCES}

Angerami, J. J., and J. O. Thomas, Studies of planetary atmospheres, 1 , The distribution of electrons and ions in the earth's exosphere, $J$. Geophys. Res., 69, 4537, 1964.

Burtis, J. W., User's guide to the Stanford VLF raytracing program, report, Radiosci. Lab., Stanford Electron. Lab., Stanford Univ., Stanford, Calif., 1973.

Cerisier, J. C., Accessibilité par propagation aux résonnances très basse fréquence dans l'ionosphere, Ann. Geophys., 23, 249, 1967.

Cerisier, J. C., Propagation perpendiculaire au voisinage de la fréquence de la résonance hybride basse, in Plasma Waves in Space and in the Laboratory, vol. 2, pp. 487-521, Edinburgh University Press, Edinburgh, 1970.

Church, S. R., and R. M. Thorne, On the origin of the plasmaspheric hiss: Ray path integrated amplification, J. Geophys. Res., 88, 7941, 1983.
Cornelleau-Wehrlin, N., J. Solomon, A. Korth, and G. Kremser, Experimental study of the relationship between energetic electrons and ELF waves observed on board GEOS, J. Geophys. Res., 90, 4141-4154, 1985.

Etcheto, J., R. Gendrin, J. Solomon, and A. Roux, A self-consistent theory of magnetospheric ELF hiss, J. Geophys. Res., 78, 8150, 1973 .

Gendrin, R., Le guidage des whistlers par le champ magnétique, Planet. Space Sci., 5, 274, 1961.

Helliwell, R. A., Whistlers and Related Ionospheric Phenomena, Stanford University Press, Stanford, Calif., 1965.

Huang, C. Y., C. K. Goertz, and R. R. Anderson, A theoretical study of plasmaspheric hiss generation, J. Geophys. Res., 88, 7927, 1983.

Kennel, C. F., and H. E. Petschek, Limit on stably trapped particle fluxes, J. Geophys. Res., 71, 1, 1966.

Kimura, I., Effects of ions on whistler-mode ray-tracing, Radio Sci., l, $269,1966$.

Lefeuvre, F., and C. Delannoy, Analysis of a random electromagnetic wave field by a maximum entropy method, Ann. Telecommun., 34, 204, 1979.

Lefeuvre, F., and R. A. Helliwell, Characterization of the sources of VLF hiss and chorus observed on GEOS-1, J. Geophys. Res., 90, 6419-6438, 1985.

Lefeuvre, F., M. Parrot, and C. Delannoy, Wave distribution functions estimation of VLF electromagnetic waves, J. Geophys. Res., $86,2359,1981$.

Lefeuvre, F., M. Parrot, L. R. O. Storey, and R. R. Anderson, Wave distribution functions for plasmaspheric hiss observed on-board ISEE-1, Tech. Note LPCE-6, CNRS/Lab. en Phys. et Chim. de l'Environ., Orléans, France, March 1983.

Lyons, L. R., and R. M. Thorne, The magnetospheric reflection of whistlers, Planet. Space Sci., 18, 1753, 1970.

Parrot, M., and F. Lefeuvre, Main characteristics of hiss observed by GEOS, in Comparative Study of Magnetospheric Systems, CNES, Toulouse, in press, 1986.

Rauch, J. L., and A. Roux, Ray tracing of ULF waves in a multicomponent magnetospheric plasma: Consequences for the generation mechanism of ion cyclotron waves, J. Geophys. Res., 87, 8191, 1982.

S-300 Experimenters, Measurements of electric and magnetic wave fields and of cold plasma parameters onboard GEOS-1: Preliminary results, Planet. Space Sci, 27, 317, 1979.

Smith, R. L., Properties of the outer ionosphere deduced from nose whistlers, J. Geophys. Res., 66, 3709-3716, 1961.

Storey, L. R. O., and F. Lefeuvre, The analysis of 6-component measurements of a random electromagnetic wave field in a magnetoplasma, I, The direct problem, Geophys. J. R. Astron. Soc., 56, $255,1979$.

Storey, L. R. O., and F. Lefeuvre, The analysis of 6-component measurements of a random electromagnetic wave field in a magnetoplasma, II, The integration kernels, Geophys. J. R. Astron. Soc., $62,173,1980$.

Thorne, R. M., and C. F. Kennel, Quasi-trapped VLF propagation in the outer magnetosphere, J. Geophys. Res., 72, 857, 1967.

Thorne, R. M., and D. Summers, Analytical solutions for resonant oblique wave growth and damping, in Comparative Study of Magnetospheric Systems, CNES, Toulouse, in press, 1986.

Thorne, R. M., E. J. Smith, R. K. Burton, and R. E. Holzer, Plasmaspheric hiss, J. Geophys. Res., 78, 1581, 1973.

Thorne, R. M., S. R. Church, and D. J. Gorney, On the origin of plasmaspheric hiss: The importance of wave propagation and the plasmapause, J. Geophys. Res., 84, 5241, 1979.

L. Cairó, Physique Mathématique Modélisation et Simulation, CNRS, 45071 Orleans Cedex 2, France.

F. Lefeuvre, Laboratoire de Physique et Chimie de l'Environnement, CNRS, 45071 Orleans Cedex 2, France.

(Received July 16, 1985; revised November 15, 1985; accepted November 18, 1985.) 